\title{
Le nom propre en lecture qualitative: de la métonymie à la métaphtonymie
}

\author{
El Mustapha Lemghari ${ }^{1, *}$ \\ ${ }^{1}$ Université Cadi Ayyad - Maroc
}

\begin{abstract}
Résumé. Le décryptage figuratif des noms propres soulève le problème de leur interprétation métonymique et/ou métaphorique. La littérature sur le sujet distingue entre les «métonymies quantitatives» et les «métonymies qualitatives", et tend, généralement, à placer les noms propres métaphoriques sous les "métonymies qualitatives». Les noms propres figuratifs sont, pour ainsi dire, basiquement métonymiques encore qu'ils soient en emploi métaphorique ou en antonomase. Il s'ensuit un certain flottement qui s'alimente, somme toute, de deux postulats aristotéliciens : d'une part, le postulat selon lequel la métonymie et la métaphore sont inconciliables en ce qu'elles s'excluent réciproquement, et d'autre part, le postulat qu'elles sont une affaire de langage, c'est-à-dire des «figures de style» ou "tropes », plutôt qu'une affaire de pensée. L'objectif de cette étude est de tenter d'élucider ce flottement à travers l'examen de quelques constructions qui jouissent d'une certaine régularité en français, en l'occurrence, 'Il y du Np chez X', 'Ça, c'est bien du Np', ' $X$ fait du Np' et ' $X$ est un Np'. On avancera l'hypothèse, conformément au modèle de la métaphore inclusive (Glucksberg \& Keysar, 1990, entre autres), que ces constructions livrent le même type d'interprétation des noms propres, pour autant qu'elles reposent sur le même mécanisme cognitif. Ce mécanisme consiste en une structure conceptuelle complexe, une métaphtonymie - pour reprendre le terme de Goossens, (1990) -, qui est la résultante de l'interaction entre métonymie et métaphore.
\end{abstract}

Mots-clés : nom propre ; métonymie ; métaphore ; massif ; comptable.

\begin{abstract}
Proper names on qualitative use: from metonymy to metaphtonymy. Decoding the figurative status of proper names raises a serious problem relative to their metonymic and/or metaphoric interpretations. The literature on the subject distinguishes between "quantitative metonymies" and "qualitative metonymies", and tends, at large, to consider metaphoric proper names as "qualitative metonymies". Thus, even on their metaphoric or antonomasic use, proper names are taken to be metonymic in character. Such a loose view follows from two main theses which can be traced back to Aristotle. On the one hand, metonymy and metaphor are irreconcilable in that they exclude each other. On the other hand, they are what the rhetoric calls "tropes", which means that they are a matter of language rather than a matter of thought. The aim of this study is to attempt to set a limit on the loose interpretations of
\end{abstract}

*El Mustapha Lemghari : e.lemghari@uca.ma 
figurative proper names in four regular constructions, that is, "Il y du Np chez X", "Ça, c'est bien du Np", "X fait du Np" and "X est un Np". We will hypothesize, in accordance with the class-inclusion model (Glucksberg \& Keysar, 1990, among others), that these constructions assign the same kind of interpretation to the figurative proper names, insofar as they are structured via the same cognitive mechanism. Such a mechanism consists in a complex conceptual structure, a metaphtonymy to use Goossens' term (1990) -, which results from the interaction between metonymy and metaphor.

Key-words: Proper noun; metonymy; metaphor; mass; countable.

\section{Introduction}

La problématique des noms propres $(\mathrm{Np})$ en emploi figuratif ${ }^{1}$ donne toujours du grain à moudre aux linguistes ${ }^{2}$. En témoigne la récente littérature sur le sujet (Flaux, 1991 et 2000 ; Gary-Prieur, 1994 ; Jonasson, 1994 et 2016 ; Kleiber, 1994a; Noailly, 2005 et 2016 ; Leroy, 2004 et 2005; Siblot et Leroy, 2000; Schnedecker, 1997; Van Langendonck, 2007, Vaxelaire, 2005, entre bien d'autres). Il est à remarquer, cependant, que les chercheurs semblent beaucoup plus intéressés par la description phénoménologique du statut métonymique ou métaphorique, ou tout autre, des $\mathrm{Np}$ que par les constructions conceptuelles sous-jacentes à leur emploi figuratif en général. On peut, dans ce sens, regretter que les études des trois dernières décennies placent l'analyse des emplois métonymiques et/ou métaphoriques des $\mathrm{Np}$ exclusivement au niveau des faits observables, qui est le niveau linguistique par excellence, c'est-à-dire au niveau de ce que la rhétorique appelle « figure de style » ou « trope ». En faisant l'impasse sur la dimension conceptuelle des $\mathrm{Np}$ en emploi figuratif, on risque de rater la nature de la relation interactionnelle qui relie dans beaucoup d'emplois la métonymie à la métaphore. C'est à l'élucidation de cette relation complexe que se dédie cette étude, élucidation d'autant plus utile que l'articulation métonymie-métaphore semble être à l'origine d'un certain flottement qui caractérise les distinctions établies dans la littérature entre emploi métonymique et emploi métaphorique des Np.

Nous nous fixons donc pour objectif de montrer que les $\mathrm{Np}$ en emploi métaphorique s'articulent sur un mécanisme conceptuel hybride, formé à la fois d'une métonymie et d'une métaphore - ce que Goossens (1990) appelle métaphtonymie (de l'anglais « metaphtonymy », formé par télescopage de métaphore et de métonymie). Chemin faisant, nous verrons comment le modèle de l'inclusion catégorielle (Glucksberg \& Keysar, 1990 et 1993 ; Glucksberg \& McGlone, 1999 ; Glucksberg \& Haught, 2006 ; Glucksberg, 2001 et 2008) permet mieux que le modèle des correspondances (Lakoff \& Johnson, 1980 et 1999 ; Lakoff \& Turner, 1989; Lakoff, 1987, entre autres), de rendre compte du décryptage figuratif des $\mathrm{Np}$ sous le double aspect de la métaphtonymie. Un peu d'eau sera également apporté au moulin de leur massivité et/ou comptabilité. Cette extension voudra tirer au clair une confusion, la confusion qui résulte de l'alignement des traits massifs et/ou comptables des $\mathrm{Np}$ en emploi métaphorique sur leurs traits en emploi métonymique.

Cette étude portera davantage sur la lecture «qualitative» des Np que sur leur lecture « quantitative $»^{3}$. En outre, l'interprétation métaphorique des $\mathrm{Np}$ n'étant pas exclusive à un type particulier de phrases, notre propos se restreint à certaines constructions qui présentent une certaine régularité en français. Ce sont les constructions : 'Il y du Np chez X', 'Ça, c'est bien du Np', 'X fait du Np' et 'X est un Np'. L'objectif final est de leur accorder une analyse unifiée.

\section{Flottement entre interprétation métonymique et métaphorique des Np figuratifs}


Les $\mathrm{Np}$ qui nous intéressent ici sont du type de ceux donnés sous (1-3):

1) a. Il y a du Chateaubriand / du Montaigne / du Beauvoir chez cet essayiste

b. Il y a du Pierre / du Marie là-dessus (Flaux, 2000)

c. Il y a du Néron dans cet homme (Flaux, 2000)

2) a. Ça, c'est bien du Mozart / du Rousseau / du Rodin / du Picasso !

b. Ça, c'est bien du Louis !

c. Ça, c'est bien du Hollande (Noailly, 2016)

3) a. En somme, Valls fait du Holland (Noailly, 2016)

b. Zlatan Ibrahimovic a, une nouvelle fois, fait du Zlatan Ibrahimovic hier soir durant la rencontre face à l'AS Saint-Etienne (cité par Jonasson, 2016).

Les énoncés (1) et (2) exemplifient les constructions 'Il y a + du Np chez X' et 'Ça, c'est bien du Np', appelées, entre autre appellations, «construction à extracteur» et « construction à présentatif » respectivement (Flaux, 2000). Les énoncés (3) exemplifient la construction ' $\mathrm{X}$ fait du Np'. Malgré leur différence morphosyntaxique, on s'accorde à dire qu'elles impliquent toutes les trois la lecture «qualitative » des Np utilisés. Mais un désaccord non moins réel semble toujours diviser les spécialistes à ce sujet. Ce désaccord émerge plus particulièrement du type d'interprétation que semble livrer chaque construction. Autrement dit, on constate qu'il n'y a pas de consensus dans la littérature sur le type de trope à l'œuvre dans chacune des trois constructions. La présente section veut éclairer quelque peu ce point en termes de la revue très rapide de quatre points de vue, celui de Gary-Prieur (1994), de Flaux (2000), de Noailly (2016) et, de Jonasson (2016).

- Pour Gary-Prieur (1994: 186), le Np quantifié est métonymique tant en lecture quantitative qu'en lecture qualitative. La différence entre les deux lectures peut être ramenée, du coup, à une sorte de clivage des entités dénotées, qui relèvent de deux ordres distincts : les entités du premier ordre, celui de la métonymie quantitative, et les entités du second ordre, celui de la métonymie qualitative: «À l'interprétation quantitative correspond une entité du premier ordre, quelque chose d'isolable et de plus ou moins concret (sonate, texte, etc.), tandis qu'à l'interprétation qualitative répond une entité du second ordre (comportement, style, manière d'être, etc.). Cette distinction requiert une précision au sujet de ce qui relie et différencie l'interprétation quantitative et l'interprétation qualitative.

Si l'on en reste au caractère $[ \pm$ concret] du référent dénoté, selon la thèse de GaryPrieur, les deux interprétations sont clairement métonymiques, puisque le lien entre le $\mathrm{Np}$ et le référent connecte deux entités, la source et la cible, dans le même domaine cognitif, en l'occurrence le domaine déployé par le Np lui-même. Soit, à titre d'exemple, les énoncés suivants :

4) On a eu du Balzac à l'examen

5) Ce texte, c'est bien du Balzac !

On s'accorde généralement à assigner une interprétation quantitative à l'énoncé (4) et une interprétation qualitative à l'énoncé (5). Toutefois, compte tenu du type de référent que chaque interprétation implique, conclure à une métonymie dans les deux énoncés est loin de faire l'unanimité des chercheurs. En cherchant à élucider la différence entre interprétation quantitative et interprétation qualitative, nous voudrions, d'une part, donner raison à GaryPrieur qui place les deux lectures dans la rubrique de métonymie et, d'autre part, montrer qu'il y a lieu de justifier les cas du flottement dans les interprétations - dans la mesure où le paradigme de l'énoncé (5) tend naturellement à entrainer la lecture métaphorique.

Pour aller vite, l'interprétation qualitative de l'énoncé (5) serait strictement métonymique, au même titre que l'interprétation quantitative de l'énoncé (4), si l'on admettait que la qualité du référent est attribuée à l'auteur Balzac. Or, il est clair que 
l'énoncé est ambigu en ce qui concerne la cible de cette attribution : la cible peut être l'auteur lui-même ou un auteur différent. De toute évidence, l'expression " ce texte », peut correspondre soit à un texte de Balzac lui-même, texte que des spécialistes de Balzac auraient trouvé et 'authentifié' comme tel, soit au texte d'un autre auteur, un essayiste par exemple, qui, par le style et les différents procédés narratifs, évoque ou rappelle les caractéristiques qualitatives de l'écriture balzacienne. Ceci nous conduit, somme toute, à examiner deux cas distincts.

(i)- Dans le premier cas, il s'agit à juste titre d'une métonymie qualitative : « métonymie », parce que l'auteur Balzac est 'pris' pour son œuvre, et « qualitative », parce que le sens de l'énoncé met en profil la qualité de cette œuvre, c'est-à-dire les propriétés qui distinguent qualitativement, dans l'univers de l'écriture littéraire, l'œuvre balzacienne d'autres œuvres. C'est cette conception, à plus forte raison, que Gary-Prieur semble vouloir mettre en valeur en proposant la distinction «métonymie quantitative » / " métonymie qualitative ». Eu égard à cette distinction, il convient de postuler deux métonymies à l'origine des deux lectures, une métonymie simple et une métonymie double ( $c f$. Rui-de Mendoza, 2014). Ainsi, la lecture quantitative de l'énoncé (4) serait structurée par la métonymie simple L'AUTEUR POUR L'EUVRE. En revanche, la lecture qualitative de l'énoncé (5) serait motivée par une métonymie double que nous formulons comme suit: L'AUTEUR POUR L'EUVRE POUR LA QUALITE DE L'CEUVRE. A son tour, la relation entre ces deux métonymies est de nature métonymique. La raison en est que la cible EUVRE dans la métonymie simple sert de source à la cible QUALITE DE L'EUVRE dans la métonymie double. On en conclut que la métonymie qualitative présuppose la métonymie quantitative. Dans ce sens, les appellations "métonymie quantitative » et "métonymie qualitative » paraissent justifiées : le lien qui relie l'œuvre à sa source est clairement métonymique ; l'œuvre de l'auteur constitue en effet le référent dans les deux interprétations. Donc, la différence - puisqu'il y en a une - entre les énoncés (4) et (5) ne tient pas dans l'œuvre ellemême mais dans les zones activées (Langacker, 1987): l'énoncé (4) active la zone matérielle et donc quantitative de l'œuvre; l'énoncé (5) en active la zone abstraite et partant qualitative.

(ii)- Dans le second cas, il s'agit de métaphore et non de métonymie qualitative. Ce constat est d'autant plus plausible que la qualité de l'œuvre est attribuée à une cible qui ne coïncide pas avec l'auteur Balzac. Autrement dit, la source et la cible de la projection métaphorique appartiennent à deux domaines cognitifs distincts. Malgré tout, l'interprétation qualitative de nature métaphorique garde un lien métonymique clair avec l'interprétation qualitative à caractère métonymique. Pour nous, c'est cette parenté 'génétique' entre le $\mathrm{Np}$ source et ses diverses extensions, quantitatives et qualitatives, qui légitime le recours à la notion de métaphtonymie. Ce point sera abordé dans la section 4 ; bornons-nous pour le moment à noter que le désaccord sur le type d'interprétation (métonymique, métaphorique, antonomasique, etc.) à accorder au paradigme de l'énoncé (5) semble participer de la confusion des deux cas distingués. Ce fait se précisera davantage dans ce qui suit.

- Flaux (2000) souscrit à l'idée d'une interprétation métonymique du Np dans les deux lectures. Mais elle double la distinction de Gary-Prieur d'un troisième type, en l'occurrence l'antonomase. Ainsi, d'une distinction bipartite des $\mathrm{Np}$ on passe à une distinction tripartite : les Np à métonymie quantitative (ex. : Je lis du Balzac; j'écoute du Mozart, etc.), les Np à métonymie qualitative (ex. : Il y a du Marie là-dessus ; ça, c'est bien du Marie !) et les Np à interprétation antonomasique ou métaphorique (ex. : Il y a du Néron dans cet homme; ça, c'est bien du Holland!). La (dis) similitude de comportement des Np dans les trois lectures a conduit Flaux $(2000: 111)$ à conclure que «[1]'antonomase se rapproche (...) de la métonymie quantitative en ce qu'elle exige un Np "notoire". Elle s'en distingue, ainsi que de la métonymie qualitative, par l'absence de la neutralisation du genre ». Malgré tout, Flaux reste indécise en ce qui concerne l'interprétation qu'il convient d'accorder aux $\mathrm{Np}$ 
métonymiques dans la construction à présentatif. D'où son interrogation « (...), la métonymie qualitative n'est-elle pas une métonymie... à titre métaphorique ? ». Et l'auteur de conclure que les constructions du type de «C'est de l'Oriane tout pur »; «Ça, c'est du Louis ! »; «C'est du Mozart!», etc., «favorisent indubitablement [le] glissement de la métonymie quantitative à la métonymie qualitative ». Nous reviendrons sur certaines des implications de la distinction tripartite de Flaux dans la section 4.

- La construction ' $X$ fait du Np', exemplifiée dans (3), n'a pas beaucoup retenu l'attention des chercheurs. Pourtant, elle est bien fréquente dans l'usage courant du français ${ }^{4}$. Les analyses récentes ${ }^{5}$ sur le sujet ne tranchent pas la question du statut métonymique et/ou métaphorique des Np utilisés. On notera tout de suite que la position de Noailly (2016) sur ce point est claire : elle place les Np dans la construction ' $X$ fait du Np' sous la rubrique des métonymies qualitatives. Cette position semble tirer son origine de la valeur péjorative que connote le $\mathrm{Np}$ dans cette construction, construction qui, selon Noailly (2016 : 114), ne donne pas lieu à l'interprétation métaphorique ou antonomasique des Np. La raison en est que la métaphore et l'antonomase «ne sont pas définitoirement péjoratives ». Expliquant la relation entre les arguments de la formule (l'argument sujet et l'argument objet de la construction) en termes de «copie» et d' "original» (i.e. l'argument sujet/(la copie) imite l'argument objet/(l'original)), elle en vient à conclure que le rapprochement du sujet et de l'objet de la formule est de l'ordre de l'analogie. Il nous semble que même en faisant fi des colorations négatives et/ou positives que peut revêtir l'énoncé dans un contexte spécifique, on n'en est pas tiré d'affaire pour autant. La preuve en est l'informativité de la formule soi-disant 'tautologique' ' $\mathrm{X}$ fait du X' (ex. : Valls fait $d u$ Valls) que Noailly (2016: 114) explique en termes de "caricature ». Selon elle, le personnage en position de ' $\mathrm{X}$ ' dans cette formule "est dit être devenu sa propre caricature ». Admettant que ce soit le cas, il est toujours difficile de voir comment un individu puisse se copier soi-même. Le problème, à notre avis, ne procède pas de l'idée de " caricature », mais du fait de ne pas ériger la "caricature », ou plus précisément le Np qui la dénote, en une catégorie inclusive (nous reviendrons plus en détails sur cette question dans la sous-section 4.2).

- Le travail de Jonasson (2016) s'inscrit dans une perspective strictement comparative. Son intérêt porte essentiellement sur la comparaison de la construction française 'faire du $\mathrm{Np}$ ' avec la construction suédoise correspondante 'Göra en $\mathrm{Np}$ ' (i.e. faire un $\mathrm{Np}$ ). L'objectif est d'élucider le pourquoi de la massivité de 'faire du Np' en français et de la comptabilité de 'Göra en Np' en suédois. Le corpus consiste en des phrases comportant le $\mathrm{Np}$ Zlatan, dont le porteur est l'international suédois Ibrahimovic Zlatan. Bien que l'auteur distingue de temps à autre les emplois métaphoriques des emplois métonymiques des $\mathrm{Np}$, elle semble adhérer à la distinction « métonymie quantitative »/ « métonymie qualitative ». En somme, elle range les $\mathrm{Np}$ à interprétation métaphorique du côté des «métonymies qualitatives ». Elle note à ce titre que dans (6), «[1] es $\mathrm{Np}$ métonymiques massifs $d u$ Whistler et $d u$ Vélasquez sont employés [ici] pour décrire un tableau qui n'est pas de Whistler ou de Vélasquez, mais dont la beauté est comparable au style de ces artistes » :

6) C'est beau comme du Whistler ou du Vélasquez (Proust, À l'ombre des jeunes filles en fleurs, 1965, cité par Jonasson, ibid. : 132)

Ce type d'emploi du $\mathrm{Np}$ est ce que Jonasson décrit comme « $\mathrm{Np}$ métonymique en interprétation métaphorique ». Tout bien considéré, le Np qui apparait dans la formule ' $\mathrm{X}$ fait du $\mathrm{Np}$ ' est nécessairement métonymique, et peut, s'il réfère aux produits / styles / comportements, etc. d'autres personnes, acquérir une interprétation métaphorique.

L'analyse de Jonasson a, cependant, une faiblesse qui semble émaner tout droit du corpus choisi. Ayant constaté que «dans les exemples français, c'est Zlatan qui fait du Zlatan et ceux qui s'y essaient échouent, alors qu'en suédois ce sont toujours d'autres qui font « un Zlatan »», Jonasson (ibid. : 133) en conclut que « [1]e Np dans göra en Zlatan est 
[donc] toujours métaphorique ». Cette conclusion a pour corollaire d'impliquer que la tournure 'tautologique' ' $\mathrm{X}$ fait du $\mathrm{X}$ ' ne livre que l'interprétation métonymique et que son interprétation, tout compte fait, est condamnée à verser dans la thèse de la «copie » et de l'« original ». Et dans la mesure où la «copie ne vaut pas l'« original » (selon Noailly), l'interprétation est forcément négative, opinion que Jonasson ne partage qu'en partie lorsqu'elle soutient, exemples à l'appui, que "[1]e sens péjoratif ne semble pourtant pas être présent dans tous exemples relevés ici de faire $d u$ Zlatan (...), et il est totalement absent dans göra en Zlatan ».

Cette mise au point rapide a pour effet de souligner le glissement d'interprétation qui caractérise les $\mathrm{Np}$ figuratifs. Il se traduit le plus souvent par un flottement autour du décryptage figuratif qu'entrainent les trois constructions. La pragma-sémantique des $\mathrm{Np}$ est appelée à s'atteler à la dissipation de ce flottement. Nous tenterons, pour notre part, de donner dans ce qui suit une analyse unifiée pour les trois constructions et partant, de montrer qu'elles sont régies par le même processus cognitif. Les $\mathrm{Np}$ recevront ainsi la même interprétation en ce qu'ils relèvent du même type de projection cognitive, la métaphtonymie.

\section{Quel modèle théorique choisir ?}

Les modèles cognitifs de la métaphore sont nombreux. De façon générale, il est possible de les ramener à deux types de modèles théoriques, les modèles maximalistes et les modèles minimalistes. Le premier type est qualifié de modèle des correspondances. Il se décline, à son tour, en différentes théories ${ }^{6}$, dont la plus célèbre est la théorie de la métaphore conceptuelle, développée par Lakoff et ses collaborateurs (1980, 1987, 1999, entre autres). Le second, probablement moins réputé que le premier, est le modèle de l'inclusion catégorielle («class-inclusion model ») (Glucksberg et ses collaborateurs 1990, 1993, 1999, entre autres). Notre choix va pour le modèle minimaliste, qui nous semble mieux approprié à la description des $\mathrm{Np}$ en emploi figuratif. Ce choix sera justifié à mesure que progressera l'analyse.

\subsection{Le modèle de la métaphore conceptuelle}

Le modèle de la métaphore conceptuelle est d'ordre analogique. Il présuppose que les projections conceptuelles reposent sur des schémas de correspondances qui associent, point pour point, des éléments du concept source à des éléments du concept cible. Ces correspondances sont systématiques, assujetties qu'elles sont au principe d'invariance (Lakoff, 1993) selon lequel la projection métaphorique préserve la topologie cognitive du concept source. Autrement dit, les correspondances entre les éléments de la source et de la cible tiennent compte de la structure du schème-image à la base du concept source (Johnson, 1987 ; Johnson \& Rohrer, 2007). A titre d'illustration, les exemples (7), (8) et (9) sont motivés par la même métaphore conceptuelle, ce que Lakoff \& Johnson (1980 et 1999) appellent L'AMOUR EST UN VOYAGE.

7) Notre relation a atteint un point de non retour

8) Notre relation a dévié du chemin tracé

9) Nous ne progressons pas ; nous tournons en rond

Cette métaphore n'est pas, à l'évidence, une expression métaphorique, mais plutôt un processus mental qui consiste à croiser deux domaines cognitifs distincts. En tant que telle, la métaphore conceptuelle désigne, en principe, la projection métaphorique elle-même, c'est-à-dire les différentes correspondances qui s'établissent systématiquement entre le domaine source VOYAGE et le domaine cible AMOUR. Le schéma des correspondances à la base de cette métaphore est communément décrit comme suit : 
- Les amoureux correspondent aux voyageurs

- La relation d'amour correspond au véhicule

- Les objectifs des amoureux correspondent à la destination des voyageurs

- Les différentes difficultés de la relation correspondent aux différents obstacles du voyage

\subsection{Le modèle de l'inclusion catégorielle}

Le modèle de l'inclusion catégorielle, quant à lui, est récalcitrant à l'idée de correspondances systématiques entre les concepts source et cible de la projection métaphorique. Du coup, il ne conçoit pas les métaphores en tant que schémas d'analogies. La métaphore, dans ce cadre, comme le rappellent Glucksberg \& Keysar (1990) à la suite de Brown (1958), est essentiellement catégorisante : elle engage le processus cognitif de catégorisation en ce sens que le concept source sert à créer une catégorie nouvelle, dont les propriétés sont attribuées au concept cible. La catégorie ainsi créée est une catégorie superordonnée, qui comprend comme membres la source et la cible de la projection conceptuelle. Etant nouvelle et essentiellement ad hoc, la catégorie super-ordonnée ne jouit pas d'une dénomination préconstruite. En conséquence, elle est identifiée sous le nom du concept source. A titre d'exemple, l'interprétation métaphorique de l'énoncé (10) se solde par la création d'une nouvelle catégorie super-ordonnée dont les propriétés référentielles sont des généralisations sur des situations caractéristiques de « désagrément », de "promiscuité», de "privation », etc. A défaut d'un nom qui encode lexicalement ce continuum de propriétés, c'est la source prison qui prête son nom à la catégorie super-ordonnée :

\section{0) Mon travail est une prison}

Qui plus est, l'entité prison n'est pas seulement le 'mandataire' de la catégorie superordonnée; elle en est aussi l'instance prototypique. Toujours est il que l'interprétation métaphorique de l'énoncé ne ressortit pas au seul fait que la source prison est l'instance exemplaire de la catégorie super-ordonnée impliquée; il faut, de plus, que la cible, en l'occurrence travail, soit incluse comme membre dans la catégorie. Ainsi, la métaphore repose essentiellement sur le groupement interactionnel de la source et de la cible de la projection. Autrement dit, la source prison et la cible travail déterminent conjointement la catégorie super-ordonnée à laquelle elles appartiennent.

Une question se pose toutefois : est-il nécessaire de parler ici d'une catégorie superordonnée nouvelle, puisque le nom prison qui la dénomme existe déjà ? On remarquera tout de suite que la langue ne reconnait pas ce nom comme catégorie super-ordonnée mais plutôt comme catégorie de base. Dans ce sens, il peut être, comme le soulignent Glucksberg \& Keyser (1990: 7), l'hyponyme de différentes catégories super-ordonnées préconstruites, telles que châtiment, établissement, etc. Mais il n'en reste pas moins vrai qu'il peut également appartenir à des catégories super-ordonnées nouvelles qui n'ont pas de noms dans la langue. Il s'ensuit que le nom prison opère dans (10) à deux niveaux distincts : au niveau de base où il désigne habituellement un 'établissement où l'on reçoit des condamnés à une peine privative de liberté', et au niveau super-ordonné où il réfère à une catégorie d'instances partageant un certain nombre de propriétés référentielles. Parmi ces membres, il se trouve que c'est l'instance de base prison qui dénomme la catégorie nouvelle.

Il reste à préciser, d'un autre côté, pourquoi c'est prison et non travail ou mariage ou tout autre membre, qui dénomme la catégorie super-ordonnée créée. L'élément de réponse apporté par la théorie de l'inclusion catégorielle repose entièrement sur la représentativité de l'instance prototypique. Soit! Mais qu'est-ce qui permet à une instance donnée d'être promue au statut d'instance prototypique ? Le modèle de l'inclusion catégorielle ne fait pas la lumière sur cette question. Toutefois, nous pouvons nous autoriser de sa conception théorique générale pour élucider le mystère. La raison résiderait, selon toute vraisemblance, 
dans la conventionnalisation de l'instance prototypique. Comparée à des instances membres comme travail et mariage, l'instance prison bénéficie d'un degré élevé de conventionalité. En conséquence, elle a plus de chance que les autres de prêter son nom à la catégorie superordonnée. Etant moins représentatives, les instances travail et mariage ne s'érigent pas naturellement en catégories super-ordonnées pour prison, comme en témoignent les énoncés douteux (11) et (12):

11) ?? Ma prison est un travail

12) ?? Ma prison est un mariage

L'inacceptabilité de ces énoncés corrobore la dimension super-ordonnée de prison. D'où par ailleurs la bonne formation de l'énoncé (10). En résumé, vu le clivage de leurs niveaux catégoriels, la catégorie super-ordonnée prison ne peut se réduire à l'instance prototypique qui la dénomme. Aussi, elle comprend un nombre théoriquement illimité de membres (i.e. travail, mariage, responsabilités, études, etc.). Mais, chose curieuse, elle peut également inclure une instance spécifique de l'instance prototypique, comme il ressort de l'exemple suivant :

\section{3) Ma prison est une (vraie) prison}

En dehors d'une interprétation tautologique, l'acceptabilité de (13) demeure principalement subordonnée à la différence du niveau taxinomique de prison en position de sujet et d'objet. Nous reprendrons ce point dans la sous-section 5.2.

\section{Application aux Np métaphtonymiques}

Le modèle de l'inclusion catégorielle est d'autant plus approprié à la description sémantique des $\mathrm{Np}$ en emploi métaphorique que ces derniers, à la différence des noms communs, ne comptent pas comme membres de catégories super-ordonnées préexistantes. Donc, les $\mathrm{Np}$ métaphoriques ont pour caractéristique essentielle de dénommer et de représenter des catégories super-ordonnées nouvelles. Il faut, par ailleurs, remarquer que le modèle de l'inclusion catégorielle ne fait pas appel à la notion de métonymie. Or, le postulat selon lequel la catégorie super-ordonnée nouvellement créée porte le nom de l'instance prototypique ne peut marcher sans toutefois présupposer l'existence d'une relation métonymique qui relie l'instance source à la catégorie super-ordonnée cible. Notre but est en effet d'expliciter cet aspect du recoupement de la métonymie et de la métaphore en emploi figuratif des $\mathrm{Np}^{7}$.

Le travail de Goossens (1990) est précurseur à cet égard, en ce sens qu'il a frayé la voie aux recherches qui abordent la métonymie et la métaphore sous l'angle de leur interaction. Goossens a constaté que la métonymie et la métaphore ne sont pas mutuellement exclusives comme le laisse penser la rhétorique. Dans bien de cas en effet, elles se combinent de façon à former ce qu'il appelle métaphtonymie. Il en distingue quatre types ${ }^{8}$. Le rôle de la métonymie dans la métaphtonymie, comme le fait remarquer Rui de Mendoza (2014 : 153), ne consiste pas à justifier la métaphore mais à y servir de substrat. En d'autres termes, l'interprétation métaphorique d'une expression métaphtonymique repose essentiellement sur la présence d'une métonymie à la base - souvent de façon implicite - de l'expression.

Force est de constater que l'interprétation figurative des $\mathrm{Np}$ dans les trois types de constructions (1-3) est structurée par le même mécanisme conceptuel. Dans chaque construction en effet le $\mathrm{Np}$, cible de la projection métaphorique, figure à l'intérieur de la source. Ce constat va presque de soi, car le modèle de l'inclusion catégorielle considère la cible et la source de la métaphore comme membres de la catégorie super-ordonnée créée. Parler de métaphtonymie pour les trois types de constructions présuppose donc que le mécanisme conceptuel à leur base est complexe. Non seulement il tient dans une addition de métaphores et de métonymies mais plus encore dans un type spécifique de matériel 
conceptuel qui est de nature strictement interactionnelle. L'inclusion de la cible de la métaphore dans la catégorie super-ordonnée conduit à penser qu'il s'agit dans ces emplois du type de métaphtonymie que Goossens appelle « metaphor within metonymy ».

On constate ainsi que le domaine source des $\mathrm{Np}$ à interprétation métaphorique est de nature métonymique. Qui plus est, il est complexe, étant constitué d'une chaine de métonymies. Dans cette perspective, l'interprétation des Np dans les énoncés (1-3) est motivée par trois métonymies. La première métonymie, LE NOM PROPRE POUR LE PORTEUR, permet d'accorder un nom à un individu, si bien que ledit individu est identifié sous ce nom (cf. Kovecses \& Radden, 1998 ; Radden \& Kovecses, 1999). Par exemple, le nom Mozart désigne l'individu Mozart, comme dans (14) :

\section{4) Le seul autre compositeur dont le génie ait approché celui de Mozart est Franz Joseph}

La seconde métonymie, L'AUTEUR POUR L'EEUVRE, opère lorsque le Np est utilisé, compte tenu de certaines conditions socio-pragmatiques, pour référer à l'œuvre du porteur (i.e. « lire du Flaubert »; « écouter du Chopin »; « acheter un Rodin, un Matisse », etc.).

Ces deux métonymies sont la condition sine qua non de la troisième métonymie, en l'occurrence L'INSTANCE PROTOTYPIQUE POUR LA CATEGORIE. Dès lors que le modèle de l'inclusion catégorielle stipule que l'instance prototypique crée, dénomme et représente la catégorie super-ordonnée, il va sans dire qu'il admet a priori l'existence d'un lien métonymique entre la catégorie et l'instance qui la représente. A ce niveau, la projection conceptuelle est de nature métonymique, s'accomplissant dans le même domaine source. En d'autres termes, le domaine source fonctionne ici comme le domaine matrice de la projection métonymique qui connecte une source, l'instance prototypique, à une cible, la catégorie super-ordonnée. Il convient de remarquer, de surcroit, que ces trois métonymies sont en rapport de présupposition selon l'ordre irréversible que voici : la métonymie L'INSTANCE PROTOTYPIQUE POUR LA CATEGORIE présuppose la métonymie L'AUTEUR POUR L'EEUVRE, qui présuppose à son tour la métonymie LE NOM PROPRE POUR LE PORTEUR.

Mais tout le problème est de savoir pourquoi la métonymie L'INSTANCE PROTOTYPIQUE POUR LA CATEGORIE ne se réduit pas exclusivement à la projection métonymique - compte tenu de ce fait que les $\mathrm{Np}$ sous (1-3), comme nous le soutenons ici, sont à interprétation métaphorique. Force est de constater que le processus, qui sous-tend les catégories superordonnées nouvellement créées, est hybride : il est à la fois métonymique et métaphorique. Ce double aspect participe de ceci que l'inclusion catégorielle peut être envisagée aussi bien comme une métaphore que comme une métonymie. Fait d'autant plus plausible que la structure de la catégorie super-ordonnée est hiérarchique, comportant comme membres des concepts basiques et d'autres subordonnés (Rosh, 1973). Il s'ensuit que le rapport du membre prototypique à la catégorie inclusive est motivé soit par la métaphore L'INCLUSION EST UNE POSSESSION soit par la métonymie UN MEMBRE D'UNE CATEGORIE POUR LA CATEGORIE.

Postuler une métaphtonymie à la base de l'inclusion catégorielle a pour avantage de montrer pourquoi les propriétés référentielles de la catégorie super-ordonnée conservent une parenté d'ordre métonymique avec l'interprétation quantitative des $\mathrm{Np}$. Ce point mérite une précision supplémentaire. Comme montré plus haut (section 2), la différence entre la métonymie quantitative et la métonymie qualitative correspond en effet à la différence des mécanismes conceptuels sous-jacents. Cependant, bien que distincts, ces mécanismes partagent une certaine filiation métonymique, pour autant qu'ils soient structurés, quoiqu'à différents niveaux de schématicité, par la métonymie L'AUTEUR POUR L'EUVRE. A vrai dire, cette métonymie est assez schématique; par conséquent, elle peut être élaborée en se doublant d'autres métonymies beaucoup plus spécifiques. Chaque métonymie a pour fonction d'activer une zone donnée de l'œuvre et partant, de livrer une interprétation particulière. Considérons, pour la clarté de l'exposé, les exemples suivants : 
15) a- Elle pourrait tout de même louer à Beyrouth puisqu'elle en avait envie, elle qui n'était pas capable de faire de différence entre Bach et Clapisson. (Proust, $U n$ Amour de Swan)

b- Ce n'est pas le cordonnier qui maudit Shakespeare ou la beauté mais au contraire celui qui continue de lire Shakespeare et ne choisit pas de faire les bottes. (Camus, l'Homme révolté)

16) a- Entendre du Wagner pendant quinze jours avec elle qui s'en soucie comme un poisson d'une pomme, ce serait gai ! (Proust, ibid.)

b- Vous me mettrez cinq Malraux et six Balzac complet en poche classique. (Cauvin, Pourquoi pas nous?)

17) a- Cette sonate, c'est bien du Bach !

b- Ce poème, c'est bien du Baudelaire !

Les Np Bach, Clapisson et Shakespeare dans les énoncés (15) ne sont pas articulés. Pourtant, ils sont en emploi métonymique. Il est évident que ces noms ne renvoient pas ici au porteurs mais à leurs œuvres. Dans les énoncés (16), les Np Wagner, Malraux et Balzac sont quantifiés, comme en témoigne leur association au partitif $d u$ et aux numératifs $c i n q$ et six ; par conséquent, ils désignent, tout comme les $\mathrm{Np}$ métonymiques nus, les œuvres des porteurs. Ces deux modes de dénoter les œuvres via les noms des porteurs se traduisent par une différence référentielle, essentiellement tributaire de la métonymie utilisée. Abstraction faite de la nature des domaines de référence (i.e. musique, littérature), l'emploi des Np nus est différent de l'emploi des Np quantifiés en ce que chaque type d'emploi active une zone spécifique de l'œuvre. Ainsi, les Np nus dans les énoncés (15) ont pour effet de référer à des œuvres saisies sous l'angle de leur intégralité. Les domaines sources sont formés par les noms eux-mêmes, et les domaines cibles sont dérivés ou se trouvent à l'intérieur des domaines sources. En bref, la métonymie à l'origine de ce type d'emploi est L'AUTEUR POUR L'EEUVRE. L'une des implications de la schématicité de cette métonymie est de présenter les référents comme neutres à la distinction massif/comptable. Ainsi par exemple «lire Shakespeare » dans (15b) peut correspondre aussi bien à une saisie homogène du référent (i.e. «lire du Shakespeare ») qu'à une saisie individualisante (i.e. «lire un/deux/trois Shakespeare »).

Dans les énoncés (16), par contre, la métonymicité des Np est tout autre : la métonymie L'AUTEUR POUR L'EEUVRE ne peut plus s'appliquer telle quelle. Elle doit être élaborée, c'està-dire, augmentée de détails à même de rendre compte de la saisie massive de (16a) et de la saisie comptable de (16b). En somme, elle se complexifie. Dit autrement, le concept cible de la métonymie simple L'AUTEUR POUR L'EUUVE sert de source pour la dérivation d'autres concepts cibles. Le concept cible EUVRE, étant neutre à la distinction massif/comptable, donne lieu à deux concepts cibles spécifiques, correspondant chacun à une saisie différente sur la dimension $[ \pm$ massif]. A cet égard, l'interprétation massive de (16a) serait motivée par la métonymie complexe L'AUTEUR POUR L'EEUVRE POUR LE REFERENT [+MASSIF], et l'interprétation comptable de (16b), par la métonymie L'AUTEUR POUR L'CEUVRE POUR LE REFERENT [+COMPTABLE].

La métonymie L'AUTEUR POUR L'EEUVRE est également à la base de la lecture qualitative des énoncés (17). Cependant, étant schématique, elle n'intervient pas directement dans la structuration de la lecture qualitative. Nous l'avons noté plus haut (section 2), la métonymie qui motive la lecture qualitative est L'AUTEUR POUR L'EUVRE POUR LA QUALITE DE L'EUVRE. Si la métonymie qualitative devait se limiter à la métonymie L'AUTEUR POUR L'EUVRE, rien en effet ne permettrait de la distinguer de la métonymie quantitative. Qui plus est, le lien métonymique entre les deux lectures est bien évident : il existe dans l'une et l'autre métonymie un invariant, en l'occurrence le concept GUVRE.

Par ailleurs, qu'est-ce qui change réellement lorsqu'au lieu d'une métonymie qualitative on a affaire à une métaphore qualitative, comme en (18a-b) ? 
18) a- Il y a du Beethoven chez ce jeune compositeur b- Ça, c'est bien du Beethoven!

Excepté le mécanisme conceptuel à la base de ces énoncés, rien ne semble changer en effet. En d'autres termes, dans la mesure où il s'agit d'une métaphore et non d'une métonymie, les propriétés qualitatives du référent ne sont pas attribuées au porteur du nom mais à une cible différente : le continuum de qualités référentielles dénotées par Beethoven n'est pas rapporté à la source Beethoven mais à d'autres compositeurs qui se laissent spécifier en termes de la situation d'énonciation. Toutefois, comme les qualités métaphoriques et les qualités métonymiques concordent parfaitement, il y a lieu d'établir une parenté métonymique entre la métaphore qualitative et la métonymie qualitative. Ceci revient, dans une certaine mesure, à postuler que la projection métaphorique dans ce type de cas repose essentiellement sur la projection métonymique. En un mot, la métaphore qualitative est métonymique à l'origine, car le faisceau de qualités, objet de l'attribution métaphorique, est généralisé sur la base de l'interprétation quantitative du $\mathrm{Np}$. Donc, elle est structurée, à un certain niveau de schématicité, en termes de la métonymie L'AUTEUR POUR L'EUVRE.

Il reste à décrire la cible de la projection métaphorique. Comme nous avons tenté de le montrer, la source de la métaphore dans le modèle de l'inclusion catégorielle est conceptuellement complexe ; elle est la résultante de la combinaison interactionnelle d'une métaphore et d'une métonymie. La cible parait tout aussi complexe que la source. La projection conceptuelle dans ce cadre ne réside pas dans des correspondances mais dans un processus d'attribution. Aussi les propriétés de la catégorie super-ordonnée sont-elles attribuées à la cible de la métaphore. A titre d'illustration, dans les exemples précités, les propriétés qui spécifient la catégorie super-ordonnée dénommée par le Np Beethoven sont attribuées à la cible de la métaphore. A première vue, la connexion ainsi établie est de nature métaphorique, d'autant plus qu'elle relie une source et une cible appartenant à deux domaines cognitifs distincts. Mais à y regarder de plus près, on s'aperçoit que cette métaphore se double d'une métonymie. En effet, selon le modèle de l'inclusion catégorielle, la catégorie super-ordonnée nouvellement créée comprend comme membres et l'instance prototypique qui la dénomme et la cible de la métaphore. En conséquence, la cible, de par son appartenance elle-même à la catégorie super-ordonnée, se laisse décrire, tout comme l'instance prototypique, sous le double aspect de la métaphtonymie.

\section{Quelques implications}

La thèse d'une analyse unifiée pour les trois types de constructions implique que le même mécanisme conceptuel est à la base de l'interprétation des $\mathrm{Np}$ utilisés. Ce postulat requiert, toutefois, une clarification au sujet de deux cas, celui de l'emploi figuratif des Np qui sont moins notoires, et celui de la soi-disant tautotologie de la tournure ' $\mathrm{X}$ fait du X'.

\subsection{La notion de notoriété}

La notion de notoriété passe dans la littérature sur les $\mathrm{Np}$ figuratifs pour critère décisif. A titre de rappel, selon Flaux (2000), à défaut d'une notoriété suffisante, les $\mathrm{Np}$ ont du mal à apparaitre en antonomase dans les constructions à extracteur et à présentatif. C'est le cas des énoncés $(1 b)$ et $(2 b)$, en comparaison à $(1 c)$ et $(2 c)$, répétés pour convenance sous $(19 a-$ b) et (20a-b) respectivement :

19) a. Il y a du Pierre / du Marie là-dessus (Flaux, 2000)

b. Ça, c'est bien du Louis !

20) a. Il y a du Néron dans cet homme (Flaux, 2000)

b. Ça, c'est bien du Hollande (Noailly, 2016) 
Pour Flaux, Pierre, Marie et Louis sont en emploi métonymique dans (19a-b), à la différence de Néron et Holland dans (20a-b) qui, eux, sont en emploi métaphorique, ou en antonomase. Le premier constat à faire est que le type de construction n'y est pour rien, puisque leur interprétation métonymique est constante aussi bien dans la construction à extracteur (19a) que dans la construction à présentatif (19b). Bref, il semblerait, à en croire Flaux, que le degré plus ou moins élevé de la notoriété des $\mathrm{Np}$ décide dans une large mesure de leur interprétation métonymique et/ou antonomasique. Le critère de la notoriété nous met donc face à un dilemme : il faut admettre que les deux types de $\mathrm{Np}$, notoires et moins notoires, ont le même statut figuratif, ou alors que les $\mathrm{Np}$ moins notoires ne sont même pas métonymiques. La seconde alternative s'avère invraisemblable, car dans l'une et l'autre construction, la référence est faite au continuum de propriétés caractéristiques des porteurs. Par contre, la première possibilité passe bien la rampe. Mais elle ne peut aller sans préciser ce qu'il faut entendre par notoriété. Distinguer l'emploi métaphorique des $\mathrm{Np}$ de leur emploi métonymique sur la foi de la dimension [ \pm notoire] donne prise à la critique. $\mathrm{Si}$ $d u$ Pierre, $d u$ Marie et $d u$ Louis sont métonymiques parce que moins notoires, on en est conduit à voir dans la notion de notoriété un critère plutôt trivial que péremptoire : on ne saurait comprendre en effet pourquoi la métonymicité des $\mathrm{Np}$ en général nécessiterait une notoriété plus large en lecture quantitative, mais une notoriété plutôt restreinte en lecture qualitative.

La notoriété demeure, cependant, un prérequis crucial à l'interprétation métonymique et/ou métaphorique des $\mathrm{Np}$. Mais il ne faut pas perdre de vue qu'elle est essentiellement une condition socio-pragmatique. Partant, elle opère tant au macro qu'au micro des contextes sociaux. Autrement dit, la notoriété ne recouvre pas seulement les contextes sociaux larges mais aussi les contextes restreints. S'il en est ainsi, rien n'empêche de la promouvoir au statut du crédo général suivant : « tout Np métonymique et/ou métaphorique est suffisamment nécessairement notoire dans le contexte culturel déployé par l'énonciation ». Selon ce principe alors, Pierre, Marie et Louis sont tout aussi notoires que Néron et Holland. Ils ne se différencient autrement que par le scope de la notoriété, qui est minimaliste pour les premiers et maximaliste pour les seconds. Il en découle, en outre, qu'ils sont structurés par le même mécanisme conceptuel : Pierre, Marie et Louis sont les instances prototypiques des catégories super-ordonnées qu'ils dénomment. Le faisceau de propriétés caractéristiques de chaque catégorie est attribué à la cible de la projection métaphorique. En d'autres termes, les qualités que Pierre, Marie et Louis instancient respectivement dans $(19 \mathrm{a})$ et $(19 \mathrm{~b})$ sont attribuées à d'autres entités'

Il est important de noter, également, que ce paradigme de $\mathrm{Np}$ s'emploie en métaphtonymie à dénoter en particulier les traits typiques et du caractère et du comportement des porteurs. Mais, contrairement aux noms des artistes, ils ne se prêtent pas à l'interprétation quantitative. Aussi sont-ils régis par une autre métonymie que L'AUTEUR POUR L'EEUVRE, en l'occurrence LE PORTEUR POUR LE CARACTERE ET/OU LE COMPORTEMENT ${ }^{10}$.

Compte tenu de la non interchangeabilité des métonymies L'AUTEUR POUR L'EUUVE et LE PORTEUR POUR LE CARACTERE ET/OU LE COMPORTEMENT, l'ambiguïté relevée à propos d'énoncés comme (21) et (22) peut enfin être tirée au clair.

21) Ça, c'est bien du Mozart ${ }^{11}$ !

22) Ça, c'est bien du Colette!

L'ambiguïté ici résulte de la double référence que présupposent les Np Mozart et Colette. En tant que $\mathrm{Np}$ d'artistes de renom, ils renvoient aux œuvres des auteurs. Mais en tant que $\mathrm{Np}$ d'individus, ils sont susceptibles de référer aux caractères et/ou aux comportements des porteurs. L'ambiguïté participe, pour le dire autrement, du fait que les $\mathrm{Np}$ d'artistes, à notoriété maximaliste ou minimaliste, impliquent les deux interprétations, quantitative et qualitative. Du coup, ils paraissent ambigus entre une référence à l'œuvre, motivée par la 
métonymie L'AUTEUR POUR L'EEUVRE et une référence au caractère et/ou au comportement, motivée par la métonymie LE PORTEUR POUR LE CARACTERE ET/OU LE COMPORTEMENT.

\subsection{La tournure ' $X$ fait du $X$ '}

La tournure ' $X$ fait du Np' vient tout juste de faire son entrée sur la scène de la linguistique française. Dans les études que nous avons rapportées plus haut (Noailly, 2016 et Jonasson, 2016), la construction 'faire du Np' est placée sous les "métonymies qualitatives ». Autrement dit, le Np y apparait comme basiquement métonymique quand bien même il se prêterait volontiers à l'interprétation métaphorique. C'est cette idée qui ressort de la position de Lecolle \& Leroy $(2006,552)$ quand elles notent à propos du paradigme de " faire du Proust» que «la métonymie se double [ici] d'une dimension métaphorique, puisqu'on peut attribuer, plus ou moins indûment, la qualité d'un auteur à un autre [...] ». En tout cas, il s'avère clair que l'appellation "métonymie qualitative » renferme une certaine ambiguïté entre emploi métonymique et métaphorique des $\mathrm{Np}$.

L'analyse unifiée proposée peut tirer le meilleur avantage de l'interaction entre métonymie et métaphore. Aussi la tournure ' $X$ fait du Np' repose-t-elle, au même titre que les tournures 'Il y a du Np' et 'Ça, c'es du Np', sur le même mécanisme métaphtonymique. La preuve peut en être donnée par le comportement significatif de la variante 'tautologique' de la formule, en l'occurrence ' $X$ fait du X'. Soit les énoncés suivants :

23) Proust imitant Saint-Simon fait du Proust (Nunez, Les Écrivains contre l'écriture, 2006, cité par Noailly, $2016: 114$ ).

24) Holland fait du Holland

25) Invité à réagir, Zlatan a donc choisi de faire du Zlatan (cité par Jonasson, 2016 : 124)

On aura reconnu dans ces exemples la formule ' $\mathrm{X}$ fait du X'. Tout de même, on conviendra d'un commun accord que ces énoncés ne sont pas de pures tautologies, tant ils sont informatifs. Or s'ils ne sont pas tautologiques, c'est-à-dire s'ils ont du sens, alors l'interprétation exclusivement métonymique des $\mathrm{Np}$ ne tient pas la route ; il faut encore l'articuler à l'interprétation métaphorique. On en est pas tiré d'affaire pour autant, puisque l'attribution métaphorique des propriétés qualitatives du référent source au référent cible n'arrange pas les choses : le Np ne semble pas sortir de sa gangue tautologique. Ce qu'il lui faut pour ce faire, c'est pouvoir référer à deux entités différentes. Autrement dit, le référent source doit être référentiellement différent du référent cible encore que l'un et l'autre soient dénotés par le même nom.

Le modèle de l'inclusion catégorielle permet dans une large mesure de rendre compte de ce phénomène. Grace à la notion de double référence (Glucksberg, 2008), il devient possible de désencombrer les énoncés de leur enveloppe tautologique. La double référence implique qu'un nom donné peut référer sur deux plans distincts, sur le plan métaphorique où il dénote la catégorie super-ordonnée dont il est le membre prototypique, et sur le plan littéral où il renvoie à l'instance particulière elle-même. Si, en emploi métaphorique du Np, la catégorie super-ordonnée dénotée n'a pas de codage linguistique, elle prend le nom de l'instance prototypique qui la représente. En conséquence, les arguments de la formule ' $\mathrm{X}$ fait du $X^{\prime}$ ne sont pas coréférentiels; ils sont plutôt membres différents de la même catégorie super-ordonnée. Dans ce sens, les énoncés (23-25) sont informatifs, car les Np en position de sujet Proust, Holland et Zlatan réfèrent littéralement aux porteurs, tandis que les Np en position d'objet $d u$ Proust, $d u$ Holland et $d u$ Zlatan renvoient métaphoriquement aux catégories super-ordonnées dénommées par ces noms mêmes.

\section{Traits massifs et/ou comptables des Np métaphtonymiques}


Peut-on postuler une distinction massif/comptable pour les $\mathrm{Np}$ à interprétation métaphtonymique à l'instar des $\mathrm{Np}$ à interprétation métonymique ? La réponse est affirmative : les $\mathrm{Np}$ métaphtonymiques apparaissent sans problème aussi bien dans le giron du massif que dans celui du comptable, comme il ressort des exemples suivants ${ }^{12}$ :

26) a- Il y a du Balzac chez cet essayiste

b- Cet essayiste est un (vrai) Balzac ; il exploite le détail à des fins expressives

27) a- Il y a du Beethoven chez ce jeune musicien

b- Ce jeune musicien est un (vrai) Beethoven ; il est très sensible aux mouvements de la nature

28) a- Ce peintre est un (vrai) Monet; il est très attentif à la variation de la lumière

b- Il y a du Monet chez ce peintre

29) a- Paul est un (vrai) Rodin

b- Il y a du Rodin chez ce sculpteur

Les Np Balzac, Beethoven, Monet et Rodin impliquent en lecture quantitative différents domaines de références qui se laissent verser, d'après la thèse de Kleiber (1994b), dans la sous-catégorie [+massif] ou la sous-catégorie [+comptable] selon qu'ils sont homogènes ou hétérogènes. Dans (26-29) cependant, ces noms sont en lecture qualitative; plus précisément, ils sont en emploi métaphtonymique. Donc, ils réfèrent aux entités du second ordre plutôt qu'à celles du premier ordre (Gary-Prieur, 1994). Il s'agit là d'un changement référentiel qui se répercute, somme toute, sur la conception de la relation massif-comptable pour les Np.

Généralement, les études sur la distinction massif/comptable ne prennent pas en ligne de compte la double référence des $\mathrm{Np}$ articulés, c'est-à-dire leur capacité à dénoter des référents métonymiques, donc quantitatifs, et des référents métaphtonymiques, partant qualitatifs. Du coup, leur sous-catégorisation qualitative [+massif] et/ou [+comptable] est calquée sur leur sous-catégorisation quantitative. Cette façon de faire a pour inconvénient de réduire les emplois massifs en (26b) et (27b) et les emplois comptables en (28a) et (29a) à de simples cas de recatégorisation. Ainsi, Balzac, Beethoven, étant référentiellement massifs en (26a) et (27a), se trouveraient convertis en comptables dans (26b) et (27b) respectivement. Inversement, Monet et Rodin, référentiellement comptables en (28a) et (29a), seraient recatégorisés en massifs dans (28b) et (29b) respectivement. Or si tel était le cas, un Balzac et un Beethoven dans (26b) et (27b) devraient impliquer un genre de médium comptabilisant, livre, sonate, entre autres. Inversement, $d u$ Monet et $d u$ Rodin en (28b) et (29b) devraient, à leur tour, présupposer une certaine massification en termes d'un « broyage » ou d'une « multiplication quantitative ${ }^{13}$, etc. Mais il n'en est rien : ces noms ne sont pas en lecture quantitative mais en lecture qualitative. Face à cette anicroche, l'heureuse issue qui peut nous tirer d'embarras serait de situer la distinction massif/comptable à deux niveaux distincts, au niveau quantitatif, où elle porte sur les référents métonymiques des $\mathrm{Np}$, et au niveau qualitatif, où elle porte sur leurs référents métaphoriques.

Compte tenu de ce clivage, la distinction massif/comptable a intérêt à prendre en compte la différence des propriétés sémantico-référentielles impliquées par chaque type de référent. En lecture quantitative, les $\mathrm{Np}$ comptables se distinguent des $\mathrm{Np}$ massifs sur la base de la structure ontologico-référentielle de leurs référents. Par exemple, Rodin et Monet renvoient dans (30) et (31) aux entités intrinsèquement comptables statue et tableau respectivement. Par contre, Bach, Beethoven et Berg réfèrent sous (32) à l'entité massive musique :

30) Il pousse des Rodin partout. (Le Parisien, 29/03/2017)

31) Un Monet, jamais exposé au public, adjugé pour 9,8 millions d'euros. (Le Monde.fr, 09/02/2012) 
32) Je sais qu'entendre Gould jouer du Bach, du Beethoven ou du Berg, ou parler à la radio, c'était écouter la musique. (Collectionscanada.gc.ca)

En lecture qualitative, cependant, la distinction massif/comptable quantitative s'annule au profit d'une distinction de second ordre en termes de l'interprétation métaphorique des $\mathrm{Np}$. Dans le modèle de l'inclusion catégorielle, les $\mathrm{Np}$ à interprétation métaphtonymique créent, dénomment et représentent des catégories super-ordonnées. Par conséquent, ils s'emploient à dénoter des propriétés référentielles abstraites, un continuum de qualités que les $\mathrm{Np}$ résument. Le changement référentiel qui s'accomplit en passant de la lecture métonymique à la lecture métaphorique a pour effet de neutraliser les traits sous-catégoriels inhérents aux $\mathrm{Np}$ en lecture quantitative. Autrement dit, les $\mathrm{Np}$ du type de Rodin, Monet, etc., perdent leurs traits quantitatifs de $\mathrm{Np}$ comptables au terme de leur métaphorisation. Mais contre toute attente, ils n'en deviennent pas massifs pour autant. En d'autres termes, il n'y a pas lieu de parler de distinction massif/comptable pour les Np métaphoriques : ils n'ont pas de sous-catégorisation propre : on ne saurait en effet leur attribuer tel ou tel trait sur la base de telle ou telle propriété, puisqu'ils consistent en un faisceau de telles propriétés.

Mais qu'en est-il alors de leurs manifestations morphosyntaxiques massives et comptables? Si le postulat de la neutralisation des traits massifs et comptables en lecture métaphorique des $\mathrm{Np}$ est plausible, la distinction massif/comptable semble se réduire, tout compte fait, à une différence de présentation du référent dénoté : une présentation massive et une présentation comptable en termes d'occurrences (Kleiber, 2011 et 2013). Ces deux moules morphosyntaxiques répondent cependant à des considérations sémantiques importantes. Il est communément admis que le partitif, à la différence du numératif, crée un certain flou qui se traduit par une référence «indistincte ». A cet égard, Jonasson (2016 : 124) a raison de suggérer que «le partitif invoque quelque chose d'incomplet, voire d'imparfait, dans sa combinaison avec un $\mathrm{Np}$ ", tandis que " l'indéfini singulier, délimitant son référent, le présente comme une entité délimitée et complète ». Transposée au modèle de l'inclusion catégorielle, cette suggestion reviendra à dire que la cible de la projection métaphorique doit être décrite de deux manières distinctes selon que la catégorie superordonnée est dénommée par le $\mathrm{Np}$ sous la forme massive 'du $\mathrm{Np}$ ' ou sous la forme comptable ' $\mathrm{X}$ est un $\mathrm{Np}$ '.

\subsection{La forme massive 'du Np}

La forme 'du Np' a pour effet d'impliquer que la cible n'est pas promue au statut de l'entité source, autrement dit, que le degré de son appartenance à la catégorie super-ordonnée est moindre à côté de la prototypicalité de l'entité source. Dire " Il y a du Np chez X», par exemple, ne fait pas de la cible ' $X$ ' l'égale de l'instance prototypique qui représente et dénomme la catégorie-super-ordonnée. D'où probablement le fait que la forme ' $\mathrm{du} \mathrm{Np}$ ' n'entraine jamais l'idée d'exemplarité. Bien que les entités cibles dans les énoncés (26a), (27a), (28b) et (29b) soient membres des catégories inclusives dénotées au même titre que les instances prototypiques, elles ne sont pas érigées en exemples. C'est là une conséquence $\mathrm{du}$ « flou » et de l' "incomplétude » qu'entraine l'emploi du partitif en général, et qui semble découler du fait que les prélèvements qu'il opère sur des masses continues, concrètes ou abstraites, demeurent sous-déterminées et donc, indistinctes. Dans ce sens, l'attribution des propriétés de la catégorie super-ordonnée à l'entité cible de la métaphore est partielle : seules quelques propriétés ou des parties de ces propriétés, sur l'ensemble des qualités de la catégorie, sont attribuées à l'entité cible.

\subsection{La forme comptable ' $X$ est un Np'}


La forme 'X est un Np' produit l'effet contraire. Elle accorde à la cible de la métaphore un degré de représentativité très proche de celui de l'instance prototypique. Mais elle n'en fait pas une instance prototypique du même calibre que l'instance qui dénomme la catégorie. Ainsi, l'idée de 'parangon', de 'modèle' qu'on associe habituellement aux Np dans les constructions ' $\mathrm{X}$ est un $\mathrm{Np}$ ' n'émerge pas de la prototypicalité de l'entité source, qui est la raison d'être de la catégorie super-ordonnée impliquée, mais de l'attribution, sous une forme «complète » des qualités de la catégorie à l'entité cible. Les implications de ce postulat sont nombreuses : limitons-nous en ici à deux, toutes deux sont au service du bienfondé du modèle de l'inclusion catégorielle.

- Tout d'abord, pour peu qu'une catégorie super-ordonnée dépourvue de codage lexical soit couramment identifiée sous un Np donné, aucune cible n'est propre à la relayer dans ce rôle - du moins synchroniquement. Autant dire que les cibles de la construction ' $\mathrm{X}$ est un $\mathrm{Np}$ ' sont des membres moins exemplaires que l'instance qui prête son nom à la catégorie. Aussi, on constate que même certains $\mathrm{Np}$ célèbres, qui peuvent servir à leur tour à dénommer et représenter des catégories super-ordonnées nouvelles, ne rechignent pas à se placer sous le toit d'autres Np. A cet égard, bien que les personnages Grandet et Harpagon incarnent chacun à sa façon un type d'avarice et profilent chacun un continuum de qualités et d'actions caractéristiques, Grandet peut plus naturellement servir de cible dans une métaphore inclusive que Harpagon. Comparons :

33) Grandet est un Harpagon

34) ?? Harpagon est un Grandet

- La seconde implication est une conséquence de la première. Elle permet de verser une autre pièce au dossier du modèle de l'inclusion catégorielle. Dès lors qu'on admet que la cible de la métaphore inclusive est membre de la catégorie super-ordonnée dénotée, on reconnait à cette catégorie, pareille en cela aux catégories taxinomiques, une structuration hiérarchique. Du coup, la structure ' $\mathrm{X}$ est un $\mathrm{Np}$ ' rentre sans problème dans le paradigme des phrases attributives qui répondent à ce que Bever et Rosenbaum (1970) appellent « Hiérarchie-Etre ». Le résultat en est que la cible de la relation hiérarchique présente une structure occurrentielle, qui peut consister en une seule ou plusieurs occurrences, comme on le voit dans (35) et (36), empruntés à Flaux (1990:43) :

35) Ce qu'il faudrait à nouveau à la France, ce sont des Jeanne d'Arc et des Napoléon

36) Moi, les Jeanne d'Arc et les Napoléon me laissent froid

On l'aura probablement assez bien montré, le trait [+comptable] qui caractérise le $\mathrm{Np}$ dans la structure 'X est un Np' ou sa variante au pluriel 'les X sont des Np' ne provient pas de la structure référentielle de la catégorie super-ordonnée dénotée, mais plus particulièrement de la structure occurrentielle de la cible de la métaphore inclusive.

\section{Conclusion}

Ce bref parcours dans le domaine des Np figuratifs a pris pour point de départ certains cas $\mathrm{du}$ flottement dans les interprétations des $\mathrm{Np}$ qui apparaissent dans une série de constructions, en l'occurrence 'Il y a du Np chez X', 'Ça, c'est bien du Np' et 'X fait du $\mathrm{Np}$ '. Les différentes recherches sur le sujet tendent, certes, à leur assigner une analyse unifiée, mais leur désaccord sur le statut, métonymique ou métaphorique, à accorder aux NP rend le projet moins heureux. Pourtant, l'idée d'une analyse unifiée pour ces constructions n'est pas triviale en soi ; elle est même nécessaire, et semble constituer à cet égard une base théorique à même de dissiper le flottement qui y 'brouille' les pistes.

Il nous semble, par ailleurs, que le flottement procède, tout bien considéré, de deux postulats aristotéliciens : d'une part, le postulat selon lequel la métonymie et la métaphore sont inconciliables en ce qu'elles s'excluent réciproquement, et d'autre part, le postulat 
qu'elles sont une affaire de langage, c'est-à-dire des «figures de style » ou «tropes », plutôt que des processus cognitifs.

L'alternative proposée ici s'inspire largement du paradigme cognitif; elle prend du coup le contrepied des analyses rhétoriques en ce sens qu'elle place, d'un côté, les phénomènes de la métonymie et de la métaphore au niveau de la pensée et les aborde, d'un autre côté, en termes de la notion de continuum. Aussi, nous reconnaissons des situations où la métonymie et la métaphore se combinent et forment, pour ainsi dire, un type particulier de projection conceptuelle, auquel nous référons ici via le terme métaphtonymie. Le but fixé donc est de décrire la structure conceptuelle des $\mathrm{Np}$ métaphtonymiques; l'hypothèse avancée est que les trois constructions reposent sur le même mécanisme cognitif et partant, génèrent le même type d'interprétation.

Fonder cette hypothèse nécessite un outillage théorique bien approprié. Nous avons opté à cet égard pour le modèle de l'inclusion catégorielle qui se prête d'autant mieux à l'analyse des $\mathrm{Np}$ que ceux-ci ne dénotent pas des catégories super-ordonnées préconstruites. Selon ce modèle, les catégories nouvellement créées, étant sans codages lexicaux, sont identifiées sous les noms de leurs instances prototypiques. Ainsi conçues et dénommées, elles comptent, entre autres membres, les instances prototypiques qui les représentent. La métaphore dans ce cadre est dite « inclusive », car elle inclut, en termes de la structuration hiérarchique de la catégorie super-ordonnée, l'entité cible et l'entité source de la projection 'attributive'. C'est là une appartenance catégorielle commune qui implique un mécanisme conceptuel complexe combinant métonymie et métaphore en une structure conceptuelle complexe, la métaphtonymie.

Enfin, pour placer un mot au sujet de la distinction massif/comptable des Np en lecture qualitative, il a fallu augmenter la série d'une quatrième construction spécialisée dans la donation comptable du Np, en l'occurrence ' $X$ est un Np'. Nous avons tenté de tirer au clair une confusion relative au statut massif et/ou comptable des $\mathrm{Np}$ métaphtonymiques. La littérature sur le sujet a pour défaut d'aligner les traits sous-catégoriels qualitatifs des $\mathrm{Np}$ sur leurs traits quantitatifs. Il s'ensuit que le statut massif et/ou comptable des $\mathrm{Np}$ ne change pas au terme du changement référentiel des référents et que, finalement, l'incompatibilité des traits quantitatifs et qualitatifs des $\mathrm{Np}$ se réduit à un phénomène de recatégorisation. Dans la mesure où les $\mathrm{Np}$ métaphtonymiques réfèrent à des catégories super-ordonnées caractérisées par un faisceau de propriétés référentielles, il s'avère inadéquat de leur postuler des traits basiques [+massif] et/ou [+comptable]. Du coup, leurs associations aux marqueurs externes de massivité et de comptabilité semblent en rester au mode d'attribution des propriétés de la catégorie à l'entité cible : une attribution «floue » et « incomplète » avec la forme massive 'du Np' et une attribution " délimitée et complète » (Jonasson, op.cit.) avec la forme comptable 'X est un Np'.

\section{Références bibliographiques}

Bever, T. G. \& Rosenbaum, P. S. (1970). Some lexical structures and Their Empirical validity. In D. Steinberg \& A. Jakobovits (éds), Semantics. Cambridge: Cambridge University Press, 586-599.

Brown, R. (1958). Words and Things. New York: Free Press.

Flaux, N. (1991). L'antonomase du nom propre ou la mémoire du réfèrent. Langue française, 92, 2645.

Flaux, N. (2000). Le nom propre et le partitif. Lexique, 15, 93-116.

Galmiche, M. (1989). Massif/comptable : de l'un à l'autre et inversement. In J. David \& G. Kleiber (éds), Termes massifs et termes comptables. Paris : Klincksieck, 63-77.

Gary-Prieur, M.-N. (1994). Grammaire du nom propre. Paris : Presses Universitaires de France. 
Gentner, D. \& Bowdle, B. F. (2001). Convention, form, and figurative language processing. Metaphor and Symbol, 16, 223-248.

Glucksberg, S. \& Keysar, B. (1990). Understanding metaphorical comparisons: Beyond similarity. Psychological Review, 97, 3-18.

Glucksberg, S. \& Keysar, B. (1993). How metaphors work. In A. Ortony (éd.), Metaphor and Thought ( $2^{\mathrm{e}}$ éd.). New York : Cambridge University Press, 401-424.

Glucksberg, S. \& McGlone, M. S. (1999). When love is not a journey: What metaphors mean. Journal of Pragmatics, 31, 1541-1558.

Glucksberg, S. (2008). How metaphors create categories - quickly. In R. Gibbs, (éd.), The Cambridge Handbook of Metaphor and Thought. New York, NY: Cambridge University Press, 67-83.

Glucksberg, S. (2001). Understanding figurative language: From metaphors to idioms. New York: Oxford University Press.

Glucksberg, S. \& Haught, C. (2006). Can Florida become like the next Florida? When metaphoric comparisons fail. Psychological Science, 17, 935-938.

Goossens, L. (1990). Metaptonymy: the interaction of metaphor and metonymy in expressions for linguistic action. Cognitive Linguistics, 3, 323-340.

Holyoak, K. \& Thagard, P. (1995). Mental leaps: analogy in creative thought. Cambridge, MA: MIT Press.

Kleiber, G. (1994a). Y a-t-il de la métaphore sous les noms propres en antonomase ?. Studia Romanica Posnaniensia, 19, 37-52.

Johnson, M. (1987). The Body in the Mind: The Bodiliy Basis of Meaning, Imagination and Reason. Chicago: Chicago University Press.

Johnson, M. \& Rohrer, T. (2007). We Are Live Creatures: Embodiment, American Pragmatism, and the Cognitive Organism. In J. Zlatev, T. Ziemke, R. Frank, R. Dirven, (éds), Body, Language, and Mind. Berlin: Mouton de Gruyter, 17-54.

Jonasson, K. (2005). La modification du nom propre dans une perspective contrastive. Langue française, 146, 67-83.

Jonasson, K. (2016). Le nom propre métonymique : massif et/ou comptable ? Le cas du nom propre Zlatan en français et en suédois. Langue française, 190, 121-138.

Kleiber, G. (1994b). Nominales. Essais de sémantique référentielle. Paris : Armand Colin.

Kleiber, G. (2011). Types de noms : le problème des occurrences. Cahiers de lexicologie, 99, 49-69.

Kleiber, G. (2013). L'opposition nom massif/nom comptable et la notion d'occurrence. Cahiers de lexicologie, 103, 85-106.

Kovecses, Z. \& Radden, G. (1998). Metonymy: Developing a cognitive linguistic view. Cognitive Linguistics, 9, 37-77.

Lakoff, G. (1987). Women, Fire and Dangerous Things: What Categories Reveal About the Mind. Chicago: University of Chicago Press.

Lakoff, G. (1993). The contemporary theory of metaphor. In A. Ortony (éd.), Metaphor and Thought, ( 2 éd.). Cambridge: Cambridge University Press, 51-202.

Lakoff, G. \& Johnson, M. (1980). Metaphors We Live By. Chicago: Chicago University Press.

Lakoff, G. \& Turner, M. (1989). More than Cool Reason: A Field Guide to Poetic Metaphor. Chicago: University of Chicago Press.

Lakoff, G. \& Johnson, M. (1999). Philosophy in the Flesh: The Embodied Mind and Its Challenge to Western Thought. New York: Basic Books. 
Langacker, R.W. (1987). Foundations of Cognitive Grammar. Vol. 1. Standford, CA: Standford University Press.

Langacker, R.W. (1991). Foundations of Cognitive Grammar; Vol. II. Descriptive Application. Standford, CA: Standford University Press.

Lecolle, M. \& Leroy, S. (2006). Collectif, massif et partition dans les figures du nom propre. In G. Kleiber, C. Schnedecker, A. Theissen (éds), La Relation 'partie-tout'. Louvain/Paris : Peeters, 543-557.

Leroy, S. (2004). De l'identification à la catégorisation : l'antonomase du nom propre en français. Louvain-La-Neuve : Peeters.

Leroy, S. (2005). L'emploi exemplaire, un premier pas vers la métaphorisation ?. Langue française, $146,84-98$.

Noailly M. (2005). Etre Chateaubriand ou rien. Langue française, 146, 39-52.

Noailly, M. (2016). Faire du Npr ou l'art du plagiat. Langue française, 190, 109-120.

Obadia, M. (1976), (éds). Grammaire 3, Les Chemins de l'expression. Le lexique. Paris : Hachette.

Pelletier, F.J. (1975). Non-Singular reference : Some Preminaries. Philosophia, 5, 4, 451-465.

Radden, G. \& Kövecses, Z. (1999). Towards a theory of metonymy. In K-U. Panther \& G. Radden (éds), Metonymy in Language and Thought. Amsterdam/Philadelphia : John Benjamins, 17-59.

Rosch, E. (1973). On the internal structure of perceptual and semantic categories. In T.E. Moore (éd.), Cognitive Development and the Acquisition of Language. New York: Academic Press, 111-144.

Ruiz de Mendoza, F. J. (2014). On the nature and scope of metonymy in linguistic description and explanation: towards settling some controversies. In J. Littlemore \& J. Taylor (éds), Bloomsbury companion to Cognitive Linguistics. London: Bloomsbury, 143-166.

Siblot, P. \& Leroy, S. (2000). L'antonomase entre nom propre et catégorisation nominale. Mots, 63, 89-104.

Schnedecker, C. (1997). Nom propre et chaînes de référence. Paris : Klincksieck.

Van Langendonck, W. (2007). Theory and Typology of Proper Names. Berlin/New-York: Mouton-De Gruyter.

Vaxelaire, J.-L. (2005). Les Noms propres : une analyse lexicologique et historique. Paris : Champion.

WEE, L. (2006). Proper names and the theory of metaphor. Journal of Linguistics, 42, 355-371.

\section{Notes}

${ }^{1}$ L'expression $N p$ figuratif sera utilisée chaque fois qu'il ne sera pas nécessaire de spécifier le statut métonymique et/ou métaphorique des $\mathrm{Np}$ dans le contexte impliqué.

${ }^{2}$ Cet article aborde les $\mathrm{Np}$ du point de vue de leur capacité à dénoter des référents autres que les porteurs. Il leur reconnait un sens référentiel; par conséquent, il prend pace, d'une façon ou d'une autre, dans le débat houleux qui disvise les linguistes sur la question de la vacuité sémantique des Np.

${ }^{3}$ La littérature sur la pragma-sémantique du Np établit, notamment depuis Gary-Prieur (1994), une distinction nette entre «interprétation quantitative» et «interprétation qualitative» des $\mathrm{Np}$ métonymiques. La différence entre les deux interprétations tient au fait que le référent en lecture quantitative est senti comme plus au moins concret; en lecture qualitative, par contre, il se prête plutôt comme abstrait. En termes de la notion de « domaine cognitif» (Langacker, 1987 et 1991), on dira que l'interprétation quantitative instancie le référent dans le domaine physique, tandis que l'interprétation qualitative l'instancie dans l'espace qualitatif. Nous reviendrons sur certaines implications de cette distinction lorsque nous présenterons le point de vue de Gary-Prieur. 


\footnotetext{
${ }^{4}$ Jonasson (2016: 122) souligne à cet égard que « Si on s'adresse aujourd'hui à Google pour chercher une construction du type faire $d u \mathrm{~Np}$, on obtient des milliers de résultats pour faire du Sarkozy, faire $d u$ Hollande, etc. ».

${ }^{5}$ Cf. Lecolle \& Leroy (2006); Jonasson (2016) ; Noailly (2016).

${ }^{6}$ Cf. Gentner \& Bowdle 2001; Holyoak \& Thagard, 1995.

${ }^{7}$ Généralement, le $\mathrm{Np}$ est métonymique et/ou métaphorique lorsqu'il est la source et non la cible d'une projection conceptuelle (Wee, 2006). A titre d'exemple, l'énoncé Paul est un cochon est, certes, métaphorique, décrivant, pour ainsi dire, l'individu Paul comme « immoral», "grossier », etc. Toujours est-il que le Np Paul n'est pas en emploi métaphorique ici, car il ne constitue pas la source mais la cible de la projection métaphorique.

${ }^{8}$ Goossens appelle les quatre métaphtonymies comme suit: « metaphor from metonymy», «metonymy within metaphor », «demetonymization inside a metaphor» et «metaphor within metonymy ».

${ }^{9}$ Dans les énoncés (19) et (20), les cibles de l'attribution métaphorique demeurent sous-déterminées tant avec les Np notoires (Néron; Holland) qu'avec les Np moins notoires (Pierre; Marie; Louis). Il s'ensuit que la création de catégories super-ordonnées est très sensible aux contextes d'énonciations. Ce fait suppose que les participants à la communication disposent d'un savoir encyclopédique, minimaliste ou maximaliste, sur les instances exemplaires des catégories créées ad hoc.

${ }^{10}$ A défaut d'un concept cible qui subsumerait des catégories basiques telles que tempérament, style, agissement, manière d'être, attitude, action, acte, etc., la métonymie LE PORTEUR POUR LE CARACTERE ET/OU LE COMPORTEMENT est déclinée en deux concepts cibles. Quoique probablement moins judicieuse, cette façon de faire entend ramener tous les traits typiques (moraux, physiques, comportementaux, etc.) du porteur à deux concepts super-ordonnés, CARACTERE et COMPORTEMENT.

${ }^{11}$ Flaux $(2000$ : 104) note à propos d'un énoncé comme (21) qu'il peut «se comprendre de deux manières, selon que $d u$ Mozart vise bien de la musique de Mozart ou bien un ou des comportements typiques de Mozart $»$.

${ }^{12}$ Certes, les emplois massifs et comptables des $\mathrm{Np}$ en lecture qualitative ne sont pas restreints aux constructions 'Il y a du Np chez X' et 'X est un Np'. Si nous nous y limitons ici, toutefois, c'est, d'une part, parce qu'elles sont systématiques en ce qu'elles ne favorisent que l'interprétation métaphorique des $\mathrm{Np}$ et, d'autre part, parce que la lecture qualitative des $\mathrm{Np}$ dans les autres types d'énoncés est très sensible aux contextes d'énonciations (voir, pour le flottement entre interprétation métonymique et interprétation métaphorique des emplois comptables ' $\mathrm{Un} \mathrm{Np}$ ', le travail de Leroy (2004)).

${ }^{13}$ Allusion est faite ici au broyeur universel (Pelletier, 1975) et au Multiplicateur (Galmiche, 1989).
} 